\title{
Endoscopic telemicrosurgery or minimally invasive robotically-assisted microsurgery for peripheral nerve repair
}

\author{
Satoshi Ichihara',2, Sybille Facca ${ }^{1}$, Frédéric Bodin ${ }^{3}$, Sarah Hendriks ${ }^{1}$, André Gay ${ }^{4}$, \\ Philippe Liverneaux ${ }^{1}$ \\ ${ }^{1}$ Department of Hand Surgery, Strasbourg University Hospital, FMTS, University of Strasbourg, 67403 Illkirch, France. \\ ${ }^{2}$ Department of Orthopaedic Surgery, Juntendo University, Tokyo 1138421, Japan. \\ ${ }^{3}$ Department of Plastic Surgery, Strasbourg University Hospital, FMTS, University of Strasbourg, 67000 Strasbourg, France. \\ ${ }^{4}$ Department of Hand Surgery, La Timone Teaching Hospital, Aix Marseille Université, 13000 Marseille, France.
}

Address for correspondence: Dr. Philippe Liverneaux, Department of Hand Surgery, Strasbourg University Hospital, FMTS, University of Strasbourg, 67403 Illkirch, France. E-mail: philippe.liverneaux@chru-strasbourg.fr

\begin{abstract}
Microsurgery comprises a variety of surgical procedures such as neurovascular anastomoses, performed under optical magnification and with fine instrumentation. While refinements have been made since its advent in the 1960s, robotics offers the potential for major technological advancement. Endoscopic telemicrosurgery is minimally invasive, robotically-assisted microsurgery. This technique removes some limitations of conventional microsurgery and enhances visual and manual dexterity. Vision is enhanced through greater magnification, three-dimensionality, and functionalization, all through an endoscopic view. Manual dexterity is improved by suppression of physiological tremor and tremor filtration, while permitting useful enhancement of movement amplitudes and tactile feedback forces. Furthermore, better endoscopic ergonomics, new hand tools and the ability for multi-manual and remote work, confer a distinct advantage. Endoscopic telemicrosurgery is already in clinical use. Some of the advantages above are incorporated into the DaVinci ${ }^{\circledR}$ robot, that is, used in brachial plexus surgery. Conventional brachial plexus surgery requires large incisions for exploration and neurotization, with its attending risks of unsightly scars, prolonged hospital stay, sepsis, and perineural adhesions that interfere with nerve regrowth. Endoscopic telemicrosurgery limits the incisions and these risks, with minimal compromise. Endoscopic telemicrosurgery, through the amplification of human capabilities may pave the way for a major advancement in the microsurgical field.
\end{abstract}

Key words:

DaVinci, endoscopy, microsurgery, robot

\section{INTRODUCTION}

Microsurgery is the surgical technique that uses both optical magnification and fine instruments in order to perform inframillimetric vascular and nerve anastomoses. The

\begin{tabular}{|l|l|}
\hline \multicolumn{2}{|c|}{ Access this article online } \\
\hline Quick Response Code: & Website: \\
\hline & www.parjournal.net \\
\cline { 2 - 2 } & \\
\hline & DOI: \\
\hline
\end{tabular}

optical magnification allows a better visualization of tissue structures than with the naked eye. The term "microsurgery", is sometimes abused because the optical magnification of surgical microscopes and surgical magnifying glasses does not exceed forty times, at best fifty times better with some supermicrosurgical microscopes. A surgical microscope must therefore be considered as binocular magnifying glass, that can visualize structures invisible to the naked eye and is not, strictly speaking, a microscope.

Microsurgical instruments provide a better repair of tissue damage than conventional instruments. Their design comes from the craft of watchmaking, whose forceps are identical. Microsurgery approaches watchmaking in which one uses a monocular magnifying glass placed over 
one eye and a fine forceps held in one hand. It differs by the use of binocular loupes and fine instruments held in both hands. The three-dimensional (3D) vision is made possible by the treatment of a shifted image for each eye, which is essential in microsurgery where the smallness of the depth of the operating field requires very precise movements.

\section{FROM MICROSURGERY TO ENDOSCOPICTELEMICROSURGERY}

Since its inception in the 1960s, microsurgery has experienced a paradoxical development. Countless surgical techniques have been described starting from digit replantation to hand transplant, through nerve repair by direct and indirect nerve grafts and neurotizations, free, and pedicled flaps, and finally to the recent applications of perforator flaps and the use of supermicrosurgery applied to less than $0.5 \mathrm{~mm}$ diameter vessels. Meanwhile, the technology itself (i.e. microscopes and instruments) has not changed in over fifty years. Microscopes have indeed evolved toward voice control screens with 3D glasses, digital image recording, and intraoperative videos, but the optical magnification has not evolved since the beginning of microsurgery. Microsurgical instruments are now made in titanium, but have remained exactly the same since their conception. Any technology experiences a revolution every half a century: it is an invariable law of industry. Hence, why has microsurgery not registered a technological leap since the 1960s? Is this due to its compartmentalization, its ignorance on the progress of other surgical disciplines? In other words, what is the future of microsurgery?

Surgery has undergone two major technological advances since the second half of the twentieth century: endoscopic surgery in the 1980s and telesurgery in the 2000s. Endoscopic surgery is the surgical technique that uses both a miniature two-dimensional (2D) camera and appropriate instruments to perform procedures by mini-invasive approaches. The operator instinctively gets an impression of $3 \mathrm{D}$ vision thanks to the micro motion of the cameras, which allows the surgeon to scan the operative field, but it is not a true $3 \mathrm{D}$ vision. Telesurgery is a surgical technique that uses a robotic remote manipulator to perform procedures without direct contact between the operator and the patient. The term robotic is an abuse of language, since the movements of the remote manipulator are performed under the direct control of the operator. Telesurgery, which suppresses the physiological tremor of the operator can combine the advantages of conventional open microsurgery with a 10 times optical magnification (up to 25 times with a digital zoom) and a 3D vision to those of endoscopic surgery thanks to instrumental and optical arms whose length allows to penetrate the surgical field by minimally invasive incisions. Telesurgery, which has many other properties, is most likely the next technological leap for the advancement of microsurgery, thanks to a new concept: telemicrosurgery.
Endoscopic telemicrosurgery and minimally invasive robotically-assisted microsurgery, which is still in its infancy, could experience a significant development in the 2020s, when a specialized robot will have been devised. The market potential is huge, especially if we think of the replacement of all conventional surgical microscopes with robotically-assisted microscopes.

\section{PROPERTIES OF ENDOSCOPIC TELEMICROSURGERY}

Only robots will cross the limits of human capabilities. Some surgical robots have already disappeared from the market $\left(\right.$ Aesope $^{\circledR}$, Zeus $\left.^{\circledR}\right)$, and others are under development (Amadeus ${ }^{\circledR}$, Newton ${ }^{\circledR}$, Gumby ${ }^{\circledR}$, etc.) including some prototypes specific to microsurgery (MSR, RAMS ${ }^{\circledR}$ ). In fact, only the DaVinci ${ }^{\circledR}$ robot is currently available on the market.

The prospect of microsurgery is to develop systems in order to enhance human capabilities of vision and to enhance manipulation of tissue repair. Ultimately, conventional microsurgery only increases two human capabilities: the 3D optical magnification thanks to binocular magnification glasses, and the manipulation of inframillimetric tissue structures by fine instruments. Endoscopic telemicrosurgery enhances other visual and manual abilities that cannot be done by conventional microsurgery [Figures 1 and 2].

\section{Magnified vision}

Optical magnification, a constitutive property of microsurgery, is possible in both conventional microsurgery and telemicrosurgery. Conventional surgery allows magnification of vision in general up to 25 times. Some supermicro surgical microscopes allow magnification up to 50 times, but the handling of tissues with ultra-fine instruments and nylon up to $14 / 0$, at the extreme limit of human capabilities, represent a barrier to the common use of supermicrosurgery. The DaVinci ${ }^{\circledR}$ robot is the only surgical robot currently available, and

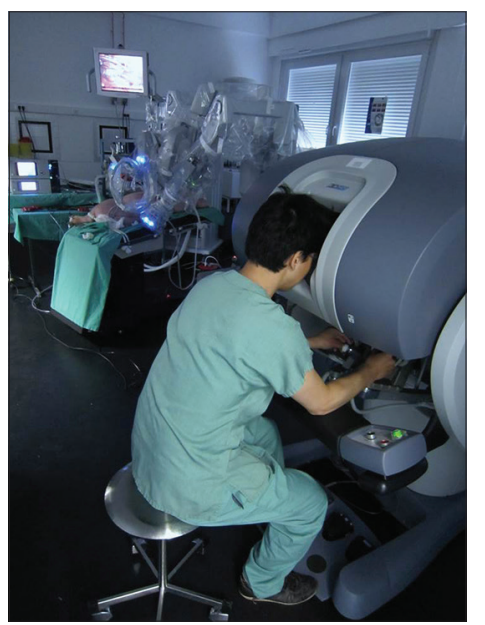

Figure 1: Installation of an endoscopic telemicrosurgical intervention in pigs. In the foreground, the operator manipulates the instruments remotely from the surgical field using the surgical console of the DaVinci ${ }^{\circledR}$ robot 


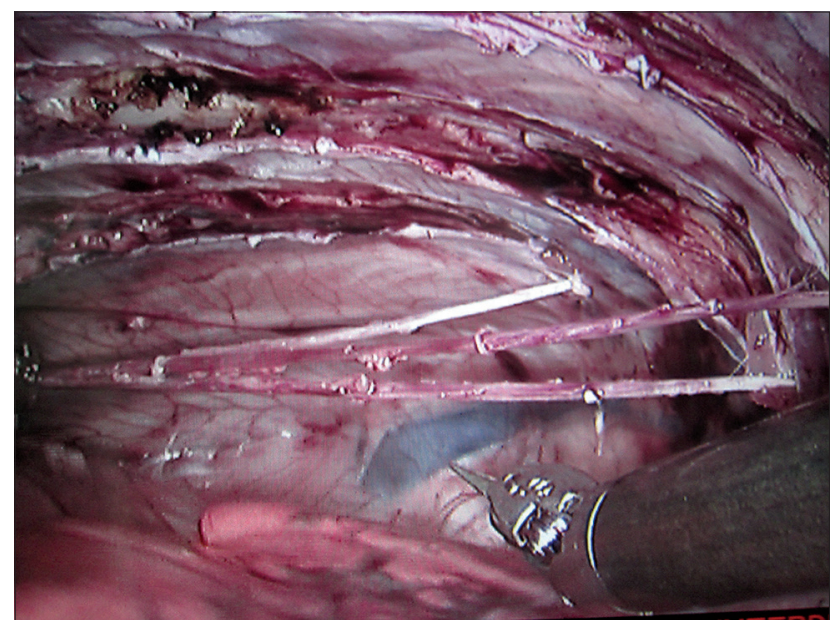

Figure 2: Intrathoracic view of three intercostal nerves in a pig harvested with the DaVinci ${ }^{\circledR}$ robot during a telemicrosurgical intervention

it allows magnification of vision up to 25 times thanks to a digital zoom. Although the current magnification of the $\mathrm{DaVinci}^{\circledR}$ robot is lower than that of conventional microscopes, it is not a limiting factor to its use in conventional microsurgery, but it is not feasible in supermicrosurgery. Considering the prospects, a specific robot to telemicrosurgery shall improve its optical magnification capacity.

\section{Three-dimensional vision}

$3 \mathrm{D}$ vision is required in microsurgery because the depth of the operating field is less than $1 \mathrm{~cm}$. 2D vision does not allow to assess acutely the position of anatomical structures and instruments into space, and can lead to tissue damage. Conventional microsurgery and telemicrosurgery allow 3D vision, a shifted image arriving to each eye in both cases, by an optical lens or CCD camera. Considering perspectives, one can mention the extreme miniaturization of cameras and obtaining a 3D vision with a single camera with methods of real-time image processing.

\section{Functionalized vision}

Vision through a conventional microscope is an external magnified vision. Various tools make it possible to see beyond the mere external appearance, revealing functional properties. ${ }^{[1]}$ Some systems enable to form an additional image that reveals microcirculation and therefore make it easier to distinguish cancerous tissue from healthy tissue by the injection of a small amount of indocyanine green, a dye tracer visualized by an infrared system. This noninvasive technique also verifies the effectiveness of a vascular anastomosis. The micro-Doppler allows to study the permeability of a vascular suture by transforming visual information of arterial or venous flow into an audible sound to the naked ear. Considering the prospects, some micro-ultrasound systems could allow to study microvascularization, but also the internal structure of peripheral nerves. This could be very useful to determine precisely the exact level of nerve transaction before performing a nerve graft of the brachial plexus for example, with respect to the method currently used (simple manual palpation of the nerve in order to perceive an internal loss of substance).

\section{Endoscopic vision}

Endoscopic surgery consists in using an endoscope within a natural or artificial body cavity. Conventional microsurgery, which uses an exoscope, does not allow endoscopic vision. The 3D camera of the DaVinci ${ }^{\circledR}$ robot can be used either as an exoscope or as an endoscope in order to practice open or endoscopic telemicrosurgery.

\section{Augmented vision}

Augmented reality consists in representing virtual data on a real image. Since the invention of this concept by Thomas Caudell in the early 1990s, augmented reality has been applied to many areas. In medicine, it naturally found technical applications using an optical devices and/or a camera: laparoscopy, ${ }^{[2]}$ arthroscopy, endoscopy, and microsurgery. Among all fields of use, the purpose of augmented reality is to simplify and to accelerate access to complex data by combining the elements of the operating field of the surgeon. Augmented reality can be applied to conventional microsurgery, but indications remain limited due to the impossibility to use endoscopy because a conventional microscope remains an exoscope and does not allow internal vision. Considering the prospects, endoscopic telemicrosurgery of the brachial plexus could evolve. From an internal view of a cavity, the anatomical structures of the brachial plexus and their relationship with other structures including vascular tissues can be difficult to identify. The registration in real time by magnetic resonance angiography images with direct intraoperative vision could act as a true anatomical global positioning system.

\section{Manual tremor filtration}

Physiological tremor in microsurgery is detrimental and unfavorable in supermicrosurgery. Telemicrosurgery makes it vanish through an interface filter, which not only improves the comfort of the surgeon, but it can also be suggestive of facilitating supermicrosurgery.

\section{Magnification of the manual movement}

The scaling of hand movement is a fundamental property in microsurgery because it increases the precision of the operator's movement. It will become indispensable in supermicrosurgery, as for example in lymphatic vessels. In the old $\mathrm{S}$ version of DaVinci ${ }^{\circledR}$ robot, the scale reached $1 / 5$. On the newer SI versions of the DaVinci ${ }^{\circledR}$ robot, the scale is reduced to $1 / 3$. The reason is that the current market is focused on telesurgery and urological laparoscopic surgery, which do not require a greater scaling. Considering the prospects, the development of a specific robot to telemicrosurgery should increase the scale of the movement up to $1 / 10$ or even more for supermicrosurgery.

\section{Magnification of manual movement amplitudes} Movements of the upper extremity and hand have limited average amplitudes due to their anatomy. For example, the normal range of motion of supination averages $180^{\circ}$. It is therefore not possible in microsurgery to make a movement of more than $180^{\circ}$ without dropping the instrument. The DaVinci ${ }^{\circledR}$ robot allows pronosupination up 
to $540^{\circ}$, repeating several times the position of the hands in the handles of the surgeon's console. The recovery of the hand position could be avoided by magnifying pronosupination in the way of a power steering system in cars. Assuming a magnification of $1 / 3$, an operator performing a pronosupination of $180^{\circ}$, could perform a pronosupination of $540^{\circ}$ in 1 time. Considering the prospects, the completion time of a vascular anastomosis could be easily decreased by performing one unique movement with the needle going from one vessel wall to the other without having to repeat the movement, especially in deep surgical fields or hard to access as in the repair of the collateral ulnar artery during thumb replantation.

\section{Magnification of the manual force feedback sensation}

The absence of force feedback or haptic sensation is often criticized in robotic surgery practiced with the DaVinci ${ }^{\circledR}$ robot. In reality, the force feedback does not exist in conventional microsurgery. Some authors have shown that the tightening sensation of a node with a $10 / 0$ nylon is perceived by a minority of individuals. ${ }^{[3]}$ In practice, the haptic sensation in conventional microsurgery is obtained indirectly by visualization of the deformity of the soft tissues in which it has acquired experience in conventional procedures where the operator directly manipulates the instruments. Unlike the DaVinci ${ }^{\circledR}$ robot, the Amadeus ${ }^{\circledR}$ robot is equipped with a device for haptic sensation, but its marketing is still confidential. The Mimic ${ }^{\circledR}$ simulator solely dedicated to training in robotic surgery is also equipped with a device for haptic sensation. Considering the prospects, if the force feedback is not currently used in conventional microsurgery and telemicrosurgery, it is not inconceivable that its magnification becomes a capital property, especially in supermicrosurgery in order to perform vascular, lymphatic, and nerve anastomoses that are currently inaccessible because of their small size.

\section{Multi-manual work}

A surgeon uses both hands to work, but an organist also uses his feet. If the DaVinci ${ }^{\circledR}$ robot has 3 instrumental arms, the same operator can only handle 2 simultaneously, even in the latest versions of the robot. The third arm is like the hand of an assistant, the operator places for example to place a retractor. In microsurgery, certain delicate gestures are performed. The advantage of the $\mathrm{DaVinci}^{\circledR}$ robot is that the third arm, unlike that of an assistant, does not tremble nor changes position. Considering the prospects, the theoretical possibility to use more than 2 instrumental arms simultaneously and by the same operator is not to be immediately eliminated on the pretext that surgeons have only used 2 of their hands till now. In fact, unconsciously, surgeons are already using their feet to activate an electrocoagulation pedal, an arthroscopic shaver or a fluoroscope. Admittedly the foot is only to activate an instrument used by hand, but it is not impossible to imagine that the order of 1 or 2 instrumental arms could be confided independently to one or two feet of a same operator. The acquisition of the independence of feet will require an equivalent learning curve to that of an organist. The assistant operational function remains to be defined.

\section{Endoscopic manual work}

Endoscopic microsurgery requires not only the introduction of a camera into a natural or artificial biological cavity, but also requires the introduction of appropriate instruments to repair damaged tissue. Conventional microsurgical instruments are not suitable for endoscopy. However, the instrumental arms of the DaVinci ${ }^{\circledR}$ robot, having a length of $50 \mathrm{~cm}$, are equipped at their termination with a miniature wrist or EndoWrist ${ }^{\circledR}$ that allows the introduction of instruments by a minimally invasive approach and which goes straight to the surgical target, as if a miniaturized operator's wrist could directly penetrate inside the body. The disadvantage remains of having performed four converging approaches. Considering the prospects, the "single port" with a miniaturized 3D endoscope equipped with instruments passing through the same flexible tube, seems to be an interesting research pathway.

\section{Augmented ergonomics}

Microsurgery is time-consuming. Interventions are long and the fatigue of the surgeon is a deleterious factor. Any factor that can improve the comfort of the surgeon can improve the quality of the intervention. In conventional microsurgery, the gaze direction of the operator does not follow a direct line between the surgeon's eye and his target. The image undergoes deflections. The consequence is that the hand-eye-head coordination is disrupted. The position of the head corresponds to a target distant to the actual target. To maintain this position, the contraction of the muscles of the neck does not match that of the actual target and can cause eyestrain and muscle fatigue. In telemicrosurgery, the gaze direction of the operator follows a direct line to his hands and the target. Paradoxically, although the surgical console is not in contact with the patient, the position of the operator's head and hand is more ergonomic than in conventional microsurgical where the operator's hands are in direct contact with the patient. In conventional microsurgery, the operative field is cluttered by the hands of the operator and his assistant. This can increase fatigue as the surgeon may have to operate in uncomfortable positions. In contrast, the operating field in telemicrosurgery is cleared, thanks to the instrumental arm length, the fineness of the EndoWrist ${ }^{\circledR}$, and especially the absence of the operator's hands.

\section{Remote working}

Conventional microsurgery requires direct contact between the patient and the surgeon. This proximity seems logical, but it is not always possible, especially when a highly specialized technical gesture is required, and no specialist surgeon is available. It is clear that at present the use of a remote expert is not current practice, but considering the prospects, in the future in the middle of a procedure requiring a very specific gesture, it may be interesting to call on a remote expert who could take control of the robot and perform a very specific task. 


\section{CLINICAL APPLICATIONS}

All properties mentioned above are not available with the DaVinci ${ }^{\circledR}$ robot. However, some of them already allow telemicrosurgical clinical applications. Among the many clinical applications, we describe its use in peripheral nerve surgery.

\section{What has been done until now?}

Our first experimental study using telemicrosurgery technique assessed the feasibility of peripheral nerve repair. ${ }^{[2]}$ Regardless of the different type of anatomical materials used (rat, pig, and human cadaver), the telemanipulator removed the physiological tremor factor during anatomical epiperineural repairs. From this experimental result, we moved to our first clinical trial to test the feasibility of the restoration of elbow flexion by Oberlin procedure using the DaVinci robot. ${ }^{[4]}$ All patients recovered elbow flexion and good functional results despite a slight difficulty in visualizing the operative field by an endoscopic approach. The development of specific retractors and instruments will probably ease these challenges. In a second clinical trial, we presented a new approach to brachial plexus surgery using mini-invasive robot-assisted surgery to perform a biopsy of an intraneural perineurioma in a 12-year-old girl. ${ }^{[5]}$ Tigan et al. ${ }^{[6]}$ also studied the surgical dissection of chronic peripheral nerve tumors using the telemicrosurgical technique to improve their results. Most recently, robot-assisted neurotization of deltoid muscle using the nerve to the long head of the triceps was described as a feasible application for the restoration of shoulder abduction after brachial plexus or axillary nerve injury. ${ }^{[7]}$ These results demonstrate that telemicrosurgery allows very safe and precise peripheral nerve repairs by counteracting physiological tremor and by improving the view of the surgical field.

\section{What are the clinical indications?}

From an anatomic positional point of view, brachial plexus injuries are the most ideal indications for telemicrosurgery. Brachial plexus injuries are caused by stretching and excessive traction on the shoulder, usually during motorcycle accidents or childbirth. We can distinguish total paralysis of the brachial plexus (most frequent lesions), paralysis of the upper C5-C6 and $\mathrm{C} 5-\mathrm{C6}-\mathrm{C} 7$ roots, and paralysis of the lower C8-T1 roots, which are rarer than total paralysis of the brachial plexus. Telemicrosurgery can also be helpful to distinguish supraclavicular and infraclavicular plexus lesions. Regardless on the type of plexus lesion, large incisions are needed either to explore the plexus or to perform neurotizations from a healthy nerve in order to reinnervate a paralyzed nerve. Apart from the unsightly appearance of these large incisions, and the lengthening of hospitalization time, these large incisions involve risks of infection and perineural adherence that interfere with the quality of nerve regrowth. Endoscopic telemicrosurgery allows interventions on peripheral nerves with minimally invasive incisions. ${ }^{[8]}$ Mantovani et al..$^{[9,10]}$ developed an effective minimally invasive approach to brachial plexus injury and showed the feasibility of using telerobotic manipulation to perform microsurgical root-to-root nerve repair of the brachial plexus with an endoscopic approach. In a cadaveric and experimental study, we already accomplished neurotization of the spinal accessory nerve to the motor branch of the musculocutaneous nerve, neurotization of the long portion of the triceps to the anterior branch of the axillary nerve, ${ }^{[1]}$ neurotization of the motor nerve fascicle of the ulnar nerve on the musculocutaneous nerve, ${ }^{[4]}$ neurolysis of the long thoracic nerve, and neurolysis of the intercostal nerve. ${ }^{[12]}$ A series of eight clinical cases of nerve damage around the shoulder girdle were operated on using the DaVinci ${ }^{\circledR}$ robot. Successful microneural repair was confirmed in all clinical studies. However, an open incision was still required. Robotic-assisted surgery of the shoulder girdle and brachial plexus is still in its early stages. ${ }^{[13]}$

\section{What are the future fields of application in nerve surgery?}

In a recent experimental study, we reported on the feasibility of robotic phrenic nerve harvest in a pig model. ${ }^{[11]}$ The advantages of using an endoscopic technique to harvest the phrenic nerve include a magnified, clear, and illuminated visualization, a better remote access incision site and an atraumatic technique. Robot-assisted neurolysis may be clinically useful for harvesting the phrenic nerve for brachial plexus reconstruction by the thoracoscopic approach.

\section{CONCLUSION}

Microsurgical techniques, magnification, and microinstruments, have not evolved since their first use in the 1960s. Endoscopic telemicrosurgery, through the amplification of human capabilities, may be the expected technological leap to introduce microsurgery in the 21 st century.

\section{REFERENCES}

I. Brahmbhatt JV, Gudeloglu A, Liverneaux P, Parekattil SJ. Robotic microsurgery optimization. Arch Plast Surg 2014;41:225-30.

2. Nectoux E, Taleb C, Liverneaux P. Nerve repair in telemicrosurgery: an experimental study. J Reconstr Microsurg 2009;25:26I-5.

3. Panchulidze I, Berner S, Mantovani G, Liverneaux P. Is haptic feedback necessary to microsurgical suturing? Comparative study of $9 / 0$ and 10/0 knot tying operated by 24 surgeons. Hand Surg 201 I; 16:1-3.

4. Naito K, Facca S, Lequint T, Liverneaux PA. The Oberlin procedure for restoration of elbow flexion with the da Vinci robot: four cases. Plast Reconstr Surg 2012; 129:707-I I.

5. Lequint T, Naito K, Chaigne D, Facca S, Liverneaux P. Mini-invasive robot-assisted surgery of the brachial plexus: a case of intraneuralperineurioma. J Reconstr Microsurg 20I 2;28:473-6.

6. Tigan L, Miyamoto H, Hendriks S, Facca S, Liverneaux P. Interest of telemicrosurgery in peripheral nerve tumors: about a series of seven cases. Chir Main 20|4;33:13-6.

7. Miyamoto $H$, Leechavengvongs S, Atik T, Facca S, Liverneaux P. Nerve transfer to the deltoid muscle using the nerve to the long head of the triceps with the da Vinci robot: six cases. J Reconstr Microsurg 20। 4;30:375-80.

8. Finley D, Sherman JH, Avila E, Bilsky M. Thorascopic resection of an apical paraspinalschwannoma using the da Vinci surgical system. J Neurol Surg A Cent Eur Neurosurg 2014;75:58-63. 
9. Mantovani G, Liverneaux P, Garcia JC Jr, Berner SH, Bednar MS, Mohr CJ. Endoscopic exploration and repair of brachial plexus with telerobotic manipulation: a cadaver trial. J Neurosurg 20 I I; I 15:659-64.

10. Garcia JC Jr, Mantovani G, Liverneaux PA. Brachial plexus endoscopy: feasibility study on cadavers. Chir Main 2012;31:7-12.

II. Porto de Melo P, Miyamoto H, Serradori T, Ruggiero Mantovani G, Selber J, Facca S, Xu WD, Santelmo N, Liverneaux P. Robotic phrenic nerve harvest: a feasibility study in a pig model. Chir Main 2014;33:356-60.

12. Latif MJ, Afthinos JN, Connery CP, Perin N, Bhora FY, Chwajol M, Todd GJ, Belsley SJ. Robotic intercostal nerve graft for reversal of thoracic sympathectomy: a large animal feasibility model. Int J Med Robot 2008;4:258-62.
13. Facca S, Hendriks S, Mantovani G, Selber JC, Liverneaux P. Robot-assisted surgery of the shoulder girdle and brachial plexus. Semin Plast Surg 20|4;28:39-44.

How to cite this article: Ichihara S, Facca S, Bodin F, Hendriks S, Gay A, Liverneaux P. Endoscopic telemicrosurgery or minimally invasive robotically-assisted microsurgery for peripheral nerve repair. Plast Aesthet Res 2015;2:220-5.

Source of Support: Nil, Conflict of Interest: None declared.

Received: 18-11-2014; Accepted: 27-04-2015 